\section{$\underset{\substack{\text { hommes } \\ \text { \& migrations }}}{ }$}

\section{Hommes \& migrations}

Revue française de référence sur les dynamiques

migratoires

1303 | 2013

Diasporas marocaines

\title{
Marocains du monde, pionniers d'une communauté transnationale
}

\section{Marie Poinsot}

\section{OpenEdition \\ Journals}

Édition électronique

URL : http://journals.openedition.org/hommesmigrations/2541

DOI : 10.4000/hommesmigrations.2541

ISSN : 2262-3353

Éditeur

Musée national de l'histoire de l'immigration

Édition imprimée

Date de publication : 1 juillet 2013

Pagination : 1

ISBN : 978-2-919040-23-0

ISSN : $1142-852 X$

\section{Référence électronique}

Marie Poinsot, « Marocains du monde, pionniers d'une communauté transnationale », Hommes \& migrations [En ligne], 1303 | 2013, mis en ligne le 21 janvier 2014, consulté le 22 septembre 2020. URL : http://journals.openedition.org/hommesmigrations/2541; DOI : https://doi.org/10.4000/ hommesmigrations.2541 


\section{MAROCAINS DU MONDE, PIONNIERS D'UNE COMMUNAUTÉ TRANSNATIONALE}

par MARIE POINSOT, rédactrice en chef

\section{Réciprocité et démocratisation des frontières}

Depuis 2003, date du dernier numéro d'Hommes \& Migrations consacré à cette migration, les flux en provenance du Maroc se sont encore diversifiés géographiquement, s'écartant des voies historiques de la France et de l'Europe pour s'orienter vers les États-Unis, le Canada, les pays du Golfe et même les pays asiatiques. Alors qu'on se demandait il y a dix ans si "on serait tenté d'y voir une diaspora en gestation", les recherches appréhendent désormais cette migration marocaine sous l'angle des dynamiques diasporiques, analysant les relations complexes entre les modalités de la dispersion et les nouveaux profils des migrants (femmes, étudiants, aventuriers, etc.) pour comprendre les positionnements d'une diaspora devenue plurielle dans les sociétés d'accueil.

N'y aurait-il pas de diasporas sans conscience et mémoire des origines ? C'est sur ce thème des constructions identitaires que les chantiers avancent, interrogeant les transmissions culturelles et la manière dont les pratiques quotidiennes les plus simples (alimentaires, corporelles, éducatives) peuvent entretenir ou non les liens des Marocains avec leur communauté d'origine. L'adaptabilité des migrants à des contextes d'accueil pluriels est déterminante dans l'évolution de leurs modèles culturels. La gestion de "l'entre-deux" identitaire multiplie les appartenances sans conflits de loyauté. Les médias communautaires et les technologies de l'information maintiennent les migrants marocains dans la "communauté transnationale" plus librement et à moindre coût. Autre dimension de la conscience des origines, celle du vieillissement des populations et de leur accompagnement ici et là-bas. Les travailleurs migrants de la première génération arrivent en effet en fin de vie, usés par des métiers pénibles et souvent démunis pour faire reconnaître leurs droits. Ce n'est pas seulement une priorité de politique publique, cela touche aussi la filiation et la mémoire des diasporas.

Les réseaux internationaux sont étudiés ici à travers la circulation des informations, les transferts financiers, la mobilité des savoirs et des qualifications qui bénéficient aux logiques diasporiques. Les Marocains dans le monde, déjà identifiés comme pionniers du développement économique du pays d'origine, en association avec les collectivités territoriales des deux rives, facilitent l'implantation des entreprises du Nord sur la base d'une double culture. Inversement, le Maroc attire aujourd'hui des compétences formées dans les pays d'accueil. Mobilité et croissance économique vont de pair à l'heure où l'Europe prône la fermeture des frontières.

Enfin, le Maroc est le seul pays du Maghreb à avoir fait un bond en avant depuis dix ans. Il s'est engagé dans une politique "diasporique" en direction de ses ressortissants à l'étranger. Avec la création d'instances de représentation des Marocains de l'étranger et l'acceptation de la double nationalité, la monarchie chérifienne détient deux manettes principales d'une citoyenneté "transnationale" qu'elle doit encore activer concrètement. Mais, pour réussir, elle devra convaincre les pays qui partagent les mêmes communautés marocaines en affichant les avantages de la réciprocité face au repli nationaliste des pays du Nord qui s'opposent à la démocratisation des frontières. 